\title{
La adaptabilidad en dos preescolares comunitarios multigrado
}

\section{Adaptability in two Multigrade Community Preschools}

\section{Steffanía Quezada Mora*}

\section{RESUMEN}

Se analiza la concreción del criterio de adaptabilidad (Tomasevski, 2004) en dos preescolares comunitarios multigrado ubicados en localidades rurales de Tulancingo, Hidalgo, México. Se observaron las clases durante una semana en cada uno de ellos, se hicieron entrevistas y algunas preguntas de reflexión a los docentes mediante bitácoras sobre algunos aspectos que consideraban para planear las actividades que proponían, las acciones que lograban y los retos que les surgieron durante su implementación. El nivel de apropiación del lenguaje escrito en niñas y niños fue fundamental para evidenciar la concreción de la adaptabilidad. Éste fue utilizado por los docentes como aspecto clave para diseñar los objetivos educativos $y$, a su vez, inflúa en la atención diferenciada hacia las niñas y los niños con mayor o menor desarrollo en este aprendizaje. En este proceso intervinieron factores internos y externos al aula que apoyaron o limitaron las adecuaciones pedagógicas propuestas. La experiencia de los docentes y los grupos pequeños de trabajo presentaron oportunidades importantes de aprendizaje para los infantes. Sin embargo, la inequidad en la infraestructura, mobiliario, materiales y la formación docente fueron limitantes.

Palabras clave: derecho a la educación, educación preescolar, educación rural, práctica pedagógica

\section{ABSTRACT}

This case study analyzes the concreteness of the adaptability criterion (Tomasevski, 2004) in two multigrade community preschools located in rural communities of Tulancingo, Hidalgo, Mexico. The classes of each were observed for a week, the teachers were interviewed and some reflection questions were made using class records focused on the main aspects they considered while planning their proposed activities, the actions they achieved, and the challenges posed during its implementation. The children's level of appropriation of the written language was fundamental to evidence to the concretion of adaptability. This was used by the teachers as a key aspect when designing the educational objectives and it also had a great influence on the differentiated attention to the children with greater or lesser development in this particular ability. All along the process, classroom's internal and external factors intervened, supporting or limiting the pedagogical adjustments proposed. The teachers' experience and small work groups presented important learning opportunities for the kids. However, the inequity in infrastructure, furniture, materials and teacher training were limiting factors in the learning process.

Key words: right to education, preschool education, rural education, pedagogical practice

\footnotetext{
"Maestría en Investigación y Desarrollo de la Educación, Universidad Iberoamericana, Ciu-
} dad de México; steffania.quezada@gmail.com 


\section{INTRODUCCIÓN}

En México la educación preescolar se oferta a través de tres tipos de servicios: general, indígena y comunitario. Estos servicios tienen elementos comunes que los articulan, como los propósitos y los contenidos curriculares que se concretan en el Programa de Educación Preescolar 2011 (PEP, 2011). No obstante, presentan características que los diferencian: el manejo de la lengua como eje transversal de la modalidad indígena y el Modelo Pedagógico Aprendizaje Basado en la Colaboración y el Diálogo (ABCD) para las llamadas escuelas comunitarias a cargo del Consejo Nacional de Fomento Educativo (Conafe).

El preescolar representa una etapa formativa de gran riqueza por el momento de desarrollo en el que se encuentran las niñas y los niños de entre 3 y 5 años de edad. Sin embargo, en México este nivel presenta problemas de equidad educativa en la existencia de recursos materiales, infraestructura y en su baja cobertura: 72.3\% (INEE, 2017a). Las limitantes anteriores se presentan de manera diferenciada según el contexto en que se encuentran las escuelas, urbano ${ }^{1}$ o rural. ${ }^{2}$

Los preescolares rurales tienden a presentar mayores carencias de equipamiento y recursos materiales (INEE, 2014), así como menores aprendizajes (INEE, 2015), mientras que 84.9\% de localidades con menos de100 habitantes no cuentan con el servicio educativo de este nivel (INEE, 2014). Durante el ciclo escolar 2015-2016, existían 89409 escuelas preescolares en México, de las cuales $27.6 \%$ eran unitarias, es decir, que un docente estaba a cargo de todos los grados existentes y las funciones directivas del centro educativo (INEE, 2017a).

La presente investigación se desarrolló en dos preescolares de tipo comunitario, es decir, los que atiende el Conafe y que constituyen casi $21 \%$ de las escuelas de este nivel en México (INEE, 2017a). Las escuelas comunitarias enfocan su atención a pequeños que viven en comunidades rurales e indígenas dispersas, caracterizadas por altos y muy altos niveles de marginación y rezago social, así como a niñas y niños migrantes que residen en campa-

\footnotetext{
${ }^{1}$ Una localidad es urbana si su población es de 2,500 o más habitantes (INEE, 2017a)

${ }^{2}$ Una localidad es rural si su población es de 1 a 2,499 habitantes (INEE, 2017a).
} 
mentos agrícolas o albergues. Se instala un centro escolar cuando se reúne un mínimo de 4 y máximo de 29 infantes en edad de cursar el nivel de educación básica correspondiente (Conafe, 2010).

La dirección del proceso de enseñanza-aprendizaje está a cargo de Líderes para la Educación Comunitaria (LEC), jóvenes de entre 16 y 29 años de edad, que cuentan al menos con secundaria concluida y ofrecen un servicio social a Conafe ${ }^{3}$ por un máximo de dos ciclos escolares (Conafe, 2010). La mayoría de ellos no tiene una formación profesional en el área educativa. Se asigna un Líder para trabajar en una comunidad y según los niveles educativos y la cantidad de niñas y niños que asisten. Normalmente, cuando el centro escolar atiende a más de 15 estudiantes, se destinan dos LEC para laborar en tales sitios. Las escuelas comunitarias son muy diversas en su operación y su organización. Su modelo pedagógico está basado en el Programa de Educación Preescolar 2011 y cuenta con un Modelo pedagógico propio, el ya mencionado ABCD. Éste encuentra sustento, como su nombre lo indica, en el diálogo y la creación de comunidades de aprendizaje centradas en aprender a aprender. La enseñanza está planteada a través de procesos de tutoría entre los diferentes actores del centro educativo y busca una articulación pedagógica y operativa entre los niveles de educación inicial y básica (Conafe, 2018).

Su principal material educativo son las Unidades de Aprendizaje Autónomo (UAA), las cuales son estrategias didácticas que buscan propiciar el aprendizaje independiente a través del diálogo y la colaboración. Son de apoyo tanto para los LEC como para los estudiantes de todos los niveles de educación básica. En ellas se brinda información general de los temas, se plantean preguntas de reflexión y un conjunto de actividades expuestas como desafíos intelectuales, así como orientaciones para que el estudiante reflexione y registre el proceso seguido en la realización de la actividad (INEE, 2017b). Por otra parte, como un material piloto distribuido en algunas entidades federativas durante el ciclo 2016-2017, a los preescolares comunitarios se les dotó del $L i$ -

\footnotetext{
${ }^{3}$ Se les otorga un apoyo económico mensual de $\$ 1200$, en promedio, y la comunidad se debe hacer cargo de su alimentación y hospedaje por el tiempo que el Líder preste el servicio. Por cada año de servicio, se le otorga una beca para que siga estudiando.
} 
bro único para aprender a leer, escribir y contar, como apoyo para que nińas y nińos aprendieran aspectos básicos de lectoescritura y pensamiento matemático.

En este artículo se despliegan los principales resultados y conclusiones de la presente investigación, que tuvo como objetivo analizar la concreción del criterio de adaptabilidad propuesto por Tomasevski (2004), desde su acepción pedagógica en dos preescolares comunitarios, a través de cinco elementos de la práctica multigrado (Santos, 2011): las relaciones interactivas, la organización de los contenidos, la distribución de los espacios y los tiempos, los materiales curriculares o recursos didácticos y los instrumentos de evaluación. Se entiende la adaptabilidad desde su acepción pedagógica como aquellos procesos que el docente realiza para que, a través del reconocimiento de la diversidad de sus estudiantes en la construcción del conocimiento, pueda distribuir de manera equitativa las oportunidades de aprendizaje para todas las nińas y los niños de un aula multigrado, con relevancia y pertinencia.

El texto está organizado de la siguiente manera: inicia presentando los referentes que le dieron estructura, seguido de la metodología, para llegar a los resultados y sus principales conclusiones, donde se incluyen posibles aspectos de las prácticas pedagógicas multigrado en los que convendría profundizar en futuros estudios.

\section{CONSTRUYENDO PUENTES PARA ANALIZAR LA ADAPTABILIDAD EN LAS ESCUELAS}

La estructura conceptual de la investigación se articula en torno a tres grandes ejes: el derecho a la educación con base en su origen en los Derechos Humanos, la equidad en educación, así como las concepciones sobre las prácticas pedagógicas multigrado como una forma de organización escolar y pedagógica específica. Cada uno de estos términos se abordará a continuación.

\section{El derecho a la Educación}

Como primera relatora especial del Derecho a la Educación (DE), cargo designado por la Organización de las Naciones Unidas (ONU) en 1998, se le encomendó a Katarina Tomasevski exami- 
nar los obstáculos que impedían la plena realización del DE. Con base en ello integró una propuesta de seguimiento del cumplimiento de los Estados para garantizar y promover el DE a través de cuatro criterios (conocidos como las 4 A's), dos de ellos dan cuenta de los derechos a la educación: asequibilidad (disponibilidad) y accesibilidad, así como los derechos en la educación: aceptabilidad y adaptabilidad, los cuales están fundamentados en los derechos humanos. La autora planteó dos aspectos clave para conceptualizarlos:

7. Las niñas y los niños son sujetos de derecho y de acuerdo con la Convención sobre los Derechos del Niño, siempre se verá por el interés superior del nińo.

8. Como características de los derechos humanos, se reconoce que éstos corresponden a todas las personas por igual (son universales), los derechos se encuentran ligados unos a otros, por lo que su respeto y protección están vinculados (son interdependientes), todos ellos son inherentes al ser humano y derivan de su dignidad (son indivisibles) y es obligación del Estado asegurar el desarrollo constructivo de ellos, evitando cualquier retroceso (son progresivos) (CNDH, 2010-2017).

En consecuencia, las 4 A's deberían estar al alcance de todas las niñas y los niños, sin favorecer la existencia de un criterio sobre otro, considerando que el desarrollo o la carencia de alguno de ellos impactará en los demás y según el criterio de progresividad estarán en perfeccionamiento en favor de proteger y garantizar mejores condiciones educativas para niñas y niños, cada una de ellas atiende distintos aspectos de los sistemas educativos que tienen implicaciones en las escuelas, como se menciona a continuación.

La asequibilidad considera dos obligaciones gubernamentales diferentes. Como derecho civil y político, requiere que el gobierno permita el establecimiento de escuelas. Mientras que el derecho a la educación como derecho social, económico y cultural demanda que el gobierno asegure que la educación gratuita y obligatoria sea asequible para todas las chicas y los chicos en edad escolar (Tomasevski, 2004). 
La accesibilidad se define de modo diferente según los niveles educativos. El gobierno se obliga a asegurar el acceso a la educación a todas las nińas y los niños en edad escolar obligatoria, pero no en el acceso al secundario ${ }^{4}$ o a la educación superior. Además, la educación obligatoria debe ser gratuita e inclusiva, mientras que las etapas posteriores pueden requerir pagos. El derecho a la educación deberá concretarse progresivamente, facilitando el acceso a la educación postprimaria en la medida en que las circunstancias lo permitan (Tomasevski, 2004).

La aceptabilidad supone garantías de calidad para la educación, estándares mínimos de salud y seguridad o requisitos profesionales para los maestros, que deben ser estipulados, dirigidos y controlados por los gobiernos. La aceptabilidad de la enseñanza se ha extendido considerablemente gracias al desarrollo del derecho internacional de los Derechos Humanos. Los derechos de los indígenas y de las minorías han priorizado la lengua de enseñanza y la prohibición de los castigos físicos ha transformado la disciplina escolar. La consideración de chicas y chicos como sujetos de derecho a la educación y de derechos en la educación ha extendido en gran medida sus límites (Tomasevski, 2004).

La adaptabilidad se centra con mayor énfasis en la escuela y su oferta. Plantea que la escuela deberá ser la que se adecue a las condiciones específicas de los alumnos, por ejemplo, el respeto y la valoración de la cosmovisión indígena en la educación, que los docentes hablen la lengua de la comunidad en la que se encuentra la escuela, que los materiales sean adecuados a esa realidad, entre otros. Se deberán considerar los contenidos y las formas de enseñanza que deben adaptarse a las características de los alumnos en el contexto cultural que se trabaja (Tomasevski, 2004).

Para ello, la adaptabilidad establece aspectos a mirar en las escuelas que den cuenta de las formas en las que éstas logran adecuarse, atender y valorar las necesidades de todas las niñas y los niños como un proceso transversal que se compone de dos vertientes principales: desde la atención a poblaciones específicas (indígenas, personas con discapacidad, migrantes, entre otros). Una segunda vertiente considera la atención a la diversidad peda-

${ }^{4}$ Es el equivalente al bachillerato en México, el cual desde 2008 es obligatorio en el país. 
gógica, considerando que los aprendices tienen diferentes estrategias y capacidades para aprender y construir su conocimiento. En ella cobra relevancia la atención diferenciada en aulas multigrado, según las necesidades de aprendizaje de los niños de diferentes grados, así como la distribución de las oportunidades de aprendizaje que se les ofrece en atención a su diversidad.

Desde el DE se reconoce que las diferencias que pueden presentarse en los sistemas educativos y, en concreto, en las escuelas respecto a la existencia y el acceso que niñas y niños tienen a los servicios educativos y a su vez a la infraestructura, el equipamiento escolar, los recursos materiales y a docentes bien formados, presenta implicaciones en las oportunidades que tienen las escuelas para adaptarse a las necesidades particulares de niñas y niños, así como en sus resultados educativos.

Ante ello se esperaría que las 4 A's estuvieran presentes en todas las escuelas, pero como criterios de análisis también permiten vislumbrar que su articulación en los sistemas educativos y en las instituciones se puede dar de distintas maneras, según el contexto, historia y necesidades particulares de los centros. Es decir, en algunos casos se podría encontrar mayor desarrollo de una $\mathrm{A}$ sobre otras, o carencias de distinta índole en las cuatro.

Al considerar estos contrastes surge la duda sobre cómo se podría apoyar el desarrollo de los criterios del DE en un contexto particular. Desde la adaptabilidad implicaría definir criterios para distribuir de manera diferenciada los recursos escolares, materiales educativos y la atención pedagógica orientados a que todos los estudiantes logren aprender. Esta investigación plantea que esta distribución sólo podría ser posible desde la equidad, es por ello que se buscó establecer vinculación teórica entre ambos conceptos.

\section{La equidad en educación}

La equidad no se puede entender si no se discuten primero dos conceptos clave: igualdad y justicia. La igualdad refiere a una relación, no a una cualidad o propiedad de la persona. Para establecer esa relación, se tendría que determinar el tipo de igualdad a la que se aspira (Bobbio, 1993, en Bolívar, 2005). El autor plantea cuatro posibilidades: igualdad para todos, igualdad para algunos, 
igualdad en todo y la igualdad entre algunos en algo. En la presente investigación se discutirá la posibilidad de tener igualdad para todos en algo, lo que invita a cuestionarse igualdad en qué. Las respuestas a esta pregunta han sido diversas, según el enfoque que se le dé (Bolívar, 2005).

Desde la perspectiva de igualdad de capacidades propuesta por Sen (1987), se plantea que identificar el tipo de igualdad al que se aspira, supondría aceptar que existen otras desigualdades o incluso algunos tipos de desigualdad deberían promoverse de manera activa por ser condición indispensable para alcanzar el tipo de igualdad que se busca. En este sentido, plantea renunciar a la búsqueda de igualdad en todo para promover relaciones equitativas o justas.

Rodríguez (2008, p. 65) plantea que desde la teoría de la justicia de Rawls (2003) "las formas legítimas o justas de desigualdad son aquellas cuya existencia puede justificarse, sea porque no es posible concebir una situación alternativa o porque esa forma de estructurar la desigualdad trae tales beneficios a la sociedad en su conjunto, que sea imprescindible para el orden social". La injusticia consistirá, simplemente, en las desigualdades que no benefician a todos. En este sentido, postula la necesidad de alcanzar la igualdad de oportunidades sobre el principio de la diferencia, beneficiando a los sectores menos favorecidos de las sociedades. Es decir, tratarlos de manera diferenciada bajo el principio de equidad con la intención de alcanzar progresivamente la igualdad.

Por su parte, Walzer (2001) introduce un nuevo elemento a considerar en el análisis sobre las formas de distribución de los bienes sociales: la dominación. Considera que el problema fundamental no es la desigualdad sino la dominación, ya que en una sociedad justa ningún bien debe servir como medio de dominación. La crítica a la dominación se basa en un principio distributivo abierto: ningún bien social $X$ ha de ser distribuido entre hombres y mujeres que posean algún otro bien $Y$ simplemente porque poseen $Y$ sin tomar en cuenta el significado de $X$. Para el autor, la igualdad es una compleja relación de personas regulada por los bienes que hacemos, compartimos e intercambiamos entre nosotros; no es una identidad de posesiones. Por lo que plantea que se requiere de una diversidad de criterios distributi- 
vos o esferas distributivas, que reflejen la diversidad de los bienes sociales. En este sentido, para que una sociedad sea justa se debe impedir que las desigualdades que existen en una esfera "contaminen" a otras. Por ello, las desigualdades educativas deben ser independientes de las desigualdades observadas en otros ámbitos como el económico o el relativo al origen cultural.

Como lo sostienen Demeuse, Baye y Doherty (2007), un sistema social equitativo no produce desigualdades injustas. De inicio, esta afirmación resulta compleja en el sentido de cuestionar sobre qué desigualdades podrían ser consideradas justas y en qué condiciones resultan aceptables y, a la vez, necesario promoverlas. Bolívar (2005) identifica que una justicia distributiva en educación debe tender a la equidad, en el sentido de repartir los medios para favorecer a los desfavorecidos, no la distribución igualitaria de recursos entre todos los alumnos. De tal forma que la equidad en educación gira en torno a la cuestión de la justicia escolar y en cómo resuelve la situación de los peor situados, en una redistribución proporcional a las necesidades.

Ante este planteamiento, el Grupo Europeo de Investigación sobre la Equidad de los Sistemas Educativos (GERESE) (2003), como se cita en Bolívar (2005, p. 3), afirma que:

un sistema educativo equitativo sería aquel que trata a todos los alumnos como iguales y que intenta favorecer una sociedad equitativa, en la que los bienes esenciales están distribuidos conforme a las reglas de la justicia y que favorece la cooperación en un plano de igualdad.

Dubet (2005), desde su postulado de la igualdad de oportunidades, entiende que la justicia escolar consistiría en velar por la equidad de las normas, en favorecer la movilidad de los alumnos y no encerrarlos precozmente en orientaciones que cierran más puertas de las que abren. Ante ello, el imperativo de la cultura común es una elección de justicia fundamental, pues preserva a los más débiles de una degradación de su situación.

En tanto, la definición de una cultura común como un bien garantizado a todos no se presenta como una opción pedagógica, sino como una "decisión de justicia", como una elección política cuyas consecuencias habría que evaluar luego en términos de 
pedagogía y de organización escolar. La prioridad de la cultura común concebida como un derecho puede permitir, entonces, todas las pedagogías posibles en función de la diversidad de sus alumnos. La escuela debe aprender a educar juntos a los alumnos diferentes (Dubet, 2005).

Al respecto, Rodríguez (2008) identifica que las aproximaciones a la equidad comparten la idea de que las personas deben tener oportunidad de desarrollar sus capacidades con independencia de las condiciones socioeconómicas y culturales de su familia de origen. A su vez, menciona que las principales diferencias surgen en lo relativo a quiénes son los que deben tener acceso a educación en cantidad y calidad similar. Al respecto considera tres posiciones principales:

a) En el caso de los que propugnan por la igualdad de oportunidades, o igualdad de acceso, subyace la idea de que son los niños de similar capacidad los que deben acceder a similares escuelas, con independencia de su situación socioeconómica. La equidad se daría, no cuando todos tengan acceso a la misma calidad educativa, sino cuando todos puedan desarrollar su potencial, con independencia del nivel sociocultural de su familia de origen. Esta posición admitiría incluso que se impartiera educación de diferente calidad, pero en función de las capacidades intelectuales, no de la clase social de origen.

b) Quienes abogan por la igualdad de trato o de medios de aprendizaje, consideran que, si bien pueden existir diferentes aptitudes individuales, todas las personas tienen la capacidad necesaria para cursar un núcleo de educación básica y que, por lo tanto, todas las personas deben tener acceso a la misma calidad de educación, al menos hasta la secundaria. No admite que deba impartirse educación de distinta calidad, ni en función de las capacidades, ni mucho menos de la clase social de la que se provenga.

c) La posición que busca la igualdad de resultados es similar a la anterior, sólo que en vez de abogar porque todos tengan acceso a educación básica de la misma calidad, admite acciones de discriminación positiva para ayudar a los más rezagados, por su condición cognitiva o socioeconómica, a alcanzar un 
nivel similar al del resto de estudiantes, para lograr que todos alcancen un logro educativo lo más igualitario posible. En esta posición se admite que puede impartirse educación de distinta calidad, si ello es necesario para ayudar a los más desfavorecidos.

Con base en las contribuciones de los autores referidos, para mirar la equidad en los preescolares comunitarios rurales se consideraron dos dimensiones: la primera asociada al momento de desarrollo en el que se encuentran las niñas y los niños que asisten a este nivel educativo, para reconocer las formas de atención adecuadas a sus necesidades que promuevan su aprendizaje, principalmente desde las prácticas pedagógicas, y la segunda relacionada con la distribución de bienes escolares a los que tienen acceso, dada la ubicación geográfica de estas escuelas.

Al mirar la igualdad y la equidad a través del criterio de adaptabilidad se busca la igualdad de resultados y la equidad en su atención, lo cual se concreta en las prácticas pedagógicas que se desarrollan en cada escuela y aulas. Tomando en cuenta que las niñas y niños poseen diferentes capacidades, viven en contextos variados y asisten a tipos de escuelas distintos, pero todos deberán acceder a un piso mínimo de formación con las mismas oportunidades de aprendizaje, cultura común, sin que éstas sean consideradas su techo máximo.

\section{Prácticas pedagógicas multigrado}

Las prácticas pedagógicas son la base de muchas acciones tendientes a favorecer, desde el aula, la atención de las necesidades educativas de niñas y niños. Por el tipo de escuela (comunitaria) en la que se desarrolló la investigación, éstas adquieren otra característica: ser multigrado, lo que las articula en un marco particular de organización escolar y pedagógica que se encuentra mediada de manera transversal por la atención a la diversidad.

La forma deorganización multigrado implica el cuestionamiento de las pautas para agrupar a niñas y niños en la escuela a través de grados, dando lugar, a su vez, a agrupaciones flexibles que consideran pautas distintas para la atención de los estudiantes, lo que se 
refleja en la disposición de los espacios y los tiempos de aula y de la institución. En estas escuelas se desarrollan situaciones educativas en la que estudiantes de diferentes edades, grados o niveles educativos alcanzados, comparten situaciones didácticas (Santos, 2011), por lo que es necesario diseñar principios pedagógicos y estrategias didácticas propias (Santos, 2007).

Pensar desde la posibilidad de una didáctica multigrado, supone un cúmulo sistemático de conocimientos teóricos que guíen y se nutran de las prácticas, construido a partir de la circunscripción de la didáctica a un campo propio de desarrollo: el grupo multigrado. Esto remite a una particular configuración de la tríada didáctica docente-alumno-saber, que busque el reconocimiento de otros elementos como el aprendizaje de los profesores, su relación con los saberes y la naturaleza, así como la circulación de éstos en el dispositivo didáctico, todos articulados a través del principio de atención a la diversidad. Se destaca la potencialidad del aprendizaje contagiado, el cual se nutre de los diferentes niveles de conocimiento y contenidos que están construyendo niñas y niños de diferentes grados y edades (Santos, 2011).

Para la estructuración de las situaciones didácticas, Santos (2011) identifica peculiaridades en distintos elementos de la práctica educativa del multigrado:

- La organización social del aula multigrado, es decir, las formas de estructurar la distribución de los alumnos en el aula, la cual pauta las posibles dinámicas grupales, la forma de convivencia, trabajo y relaciones entre los alumnos.

- Las relaciones interactivas. Refieren al lugar del alumno y del docente y las posibles relaciones que se establecen entre los alumnos y entre éstos y el docente. Implica las formas en las que se establece la comunicación, los vínculos afectivos, el fomento de la autonomía, la confianza y la delegación de responsabilidades.

- La distribución de los espacios y los tiempos, típicamente prescritos por la didáctica en términos de organización, estructuración y planificación en aulas multigrado.

- Los materiales curriculares o recursos didácticos, resultan clave porque pueden ser tomados como punto de partida en la 
prescripción de materiales didácticos.

- La organización de los elementos anteriores, a través de las secuencias de actividades didácticas, la manera de encadenar diferentes actividades a lo largo de una unidad didáctica, con fines explícitos vinculados a los aprendizajes.

- Los instrumentos de evaluación.

- Organización de los contenidos en sus diferentes modalidades, según el modelo curricular y didáctico que esté en juego. En las aulas multigrado se presenta como la escena fundante de lo didáctico, en tanto que es a partir de este elemento que se desencadena el resto (tiempo, espacios, recursos, organización de clase, relaciones interactivas, evaluación), de acuerdo con el grado de complejidad que implica el trabajo simultáneo de varios grados integrando un mismo grupo.

En torno a los saberes y la forma en la que éstos se van traduciendo en contenidos educativos, se moldea y se da sentido a los procesos de enseñanza-aprendizaje, los cuales se encuentran pautados por la intencionalidad del docente. Para explicar este proceso de concreción, Santos (2011) plantea cuatro niveles o hábitats del saber, que refieren a los procesos mediante los cuales el conocimiento científico, como punto de partida, se va convirtiendo en un contenido escolar a enseñar, a aprender y a ser utilizado en contextos fuera del escolar, son los siguientes: 1) el ámbito de producción, 2) los saberes a enseñar, el ámbito de lo curricular, 3) saberes enseńados, 4) los saberes aprendidos.

Sobre el segundo, Santos (2007, p. 79) menciona que la presencia y la circulación de los saberes multigrado constituye:

la producción de ideas acerca de la generación de marcos abiertos de trabajo, el acceso libre al saber por parte de niños de diferentes grados, las posibilidades de interacción entre ellos en torno al saber y las diferentes maneras de romper con la estructura de escuela graduada en términos didácticos... Una circulación que supone abrir las formalidades de los grados escolares, para que los saberes fluyan de acuerdo a criterios epistémicos más que psicológicos, de acuerdo a las circunstancias más que a las formalidades. 
El mismo autor plantea que la articulación didáctica para los grupos multigrado tiene efectos sobre la forma de estructurar los procesos de enseñanza, con la intención de atender la diversidad desde lo didáctico, lo cual no significa que sea exclusiva para este tipo de organización escolar, sino que podría ser deseable para cualquier grupo, ya que dentro de las aulas graduadas también se presenta una enorme diversidad en los niveles y características de apropiación del conocimiento de niñas y niños.

Con relación en lo planteando, y a la luz de las teorías del aprendizaje desde una perspectiva constructivista, se reconocen dos aspectos clave para el desarrollo infantil: por un lado, la interacción de personas con distintas capacidades y conocimiento $y$, por otro, las situaciones del entorno y educativas en las que se ven implicados. Sobre la primera, resulta fundamental que niñas y niños pequeños o que tienen menos experiencia se encuentren implicados en tareas comunes con los individuos más capaces; ya que cuando los primeros actúan junto con una persona más 184 capaz, puede llegar a hacer algunas cosas que no podría hacer en caso de estar solo (Bassedas, Huguet y Sole, 2006), favoreciendo el aprendizaje contagiado.

Por otra parte, las situaciones a las que se enfrentan nińas y niños son vehículos de significados, que les dan contexto, de tal suerte que no se asimilan objetos "puros", sino situaciones en las cuales los objetos desempeñan ciertos papeles y no otros (Piaget y García, 1982, en Ferreiro, 1999). Reconociendo que conforme se avanza en el aprendizaje los significados construidos se irán modificando con la intención de reconstruirlos de manera más autónoma.

Estas experiencias son importantes porque permiten a niñas y niños establecer relaciones entre los objetos y diferentes situaciones que son base para hacer nuevas construcciones y ampliar el campo de conocimientos. Esto implica que desde las acciones educativas se utilicen los problemas, las situaciones, los materiales y los escenarios que los pequeños encuentran en su vida diaria para identificarlos y analizarlos. También se deberá tener en cuenta el contexto sociocultural de niñas y nińos y planificar la práctica educativa para ofrecerles las experiencias que tal vez no hayan vivido en su ambiente familiar y cultural (Bassedas, $\mathrm{Hu}-$ guet y Sole, 2006). 
Al establecer una relación conceptual explícita entre el criterio de adaptabilidad, la equidad y las prácticas pedagógicas multigrado, se visualizaron aspectos clave que pueden favorecer el aprendizaje de niñas y niños a través de la valoración de sus diferentes formas de construcción del aprendizaje, así como las interacciones generadas entre los saberes de los miembros de un grupo multigrado, sin importar su edad. Esto permitió plantear una metodología de acercamiento a dos preescolares comunitarios, así como ejes para analizar la información recabada.

\section{PARA MIRAR LA ADAPTABILIDAD EN DOS PREESCOLARES}

Con la intención de responder y comprender cómo se desarrolla el criterio de adaptabilidad en las prácticas pedagógicas multigrado de dos preescolares comunitarios, el trabajo de campo se desarrolló durante el ciclo 2016-2017. En ambas escuelas se observó el desarrollo de cinco sesiones de clase en cada aula, se entrevistó a los LEC y a padres de familia. Además, los LEC llenaron bitácoras de las unidades de aprendizaje observadas, las cuales tuvieron la intención de brindarles un espacio intencionado de reflexión sobre su práctica, respecto a lo que había planeado y lo logrado durante sus clases a través de siete ${ }^{5}$ preguntas.

Los instrumentos utilizados se enriquecieron con la información que se recolectó a través del diálogo constante con los LEC, alimentado por las situaciones que se observaban en el aula y las propias inquietudes que surgían en ambas partes. Adicionalmente, la perspectiva de los padres de familia permitió conocer sus inquietudes y expectativas sobre la formación de sus hijos en el preescolar. En conjunto, estos instrumentos abonaron a conocer las interacciones cotidianas que se producían en el aula, así como a recuperar su percepción sobre los retos que identificaban los LEC para trabajar en grupos multigrado de preescolar.

Con base en la información del trabajo de campo y los referentes presentados se construyeron tres categorías de análisis:

\footnotetext{
${ }^{5}$ ¿Qué intención tenía la actividad?, ¿qué hicieron las niñas y los niños durante la actividad?, ¿cómo intervino usted durante la actividad?, ¿cómo planeó lograr su objetivo?, ¿qué considera que logró?, ¿cuáles fueron las dificultades que se le presentaron?, ¿qué podría mejorar de esta actividad?
} 
- Equidad en la atención educativa multigrado como la construcción de oportunidades de enseñanza y de aprendizaje particulares y diferenciadas, orientadas a que todas las niñas y los niños de un grupo logren los aprendizajes propuestos, sin importar su contexto sociocultural, su edad y sus habilidades o capacidades personales (Bolívar, 2005; Rodríguez, 2008).

- Relevancia de los aprendizajes, entendida como las acciones escolares orientadas a dotar de significado a las experiencias educativas de niñas y niños y que éstas sean fundamentales para que puedan seguir aprendiendo (Santos, 2011).

- Pertinencia cultural de la atención educativa multigrado, como el reconocimiento a través de los contenidos y las prácticas escolares de aquellos aspectos que, desde lo global, lo nacional y lo local, son considerados como lo mejor y lo más valioso, durante un momento histórico determinado y en función de las expectativas a futuro de determinada formación (Santos, 2011).

Tales categorías permitieron mirar de manera transversal cinco elementos de la práctica multigrado: las relaciones interactivas, la organización de contenidos, la distribución de los espacios y los tiempos, los materiales curriculares o recursos didácticos y los instrumentos de evaluación.

\section{LA CONCRECIÓN DEL CRITERIO DE ADAPTABILIDAD EN DOS PREESCOLARES COMUNITARIOS}

En este apartado, de manera inicial, se plantean algunos aspectos de contexto que dan cuenta, principalmente, de los criterios de disponibilidad y accesibilidad del DE en recursos humanos y materiales con los que contaban los preescolares visitados. Estos criterios tienen una importante implicación en la concreción de la adaptabilidad, como aspectos que fuera y dentro del aula favorecieron o limitaron su desarrollo. En un segundo momento se presentan algunos hallazgos y reflexiones en torno al papel central que se identificó en el desarrollo de la lectoescritura, como objeto y medio de aprendizaje. Los resultados se presentan a manera de contraste, guardando el anonimato de los informantes, mediante el uso de las letras A y B para referirlos. 
El preescolar A no contaba con instalaciones propias; los padres de familia rentaban un cuarto ubicado dentro de la casa de una señora de la comunidad. El espacio era muy reducido para realizar las actividades escolares y no tenía sillas y mesas para los nueve nińos del grupo. El B se integraba de dos aulas propias, con salones amplios en relación con la cantidad de niños del grupo (15 infantes) y contaban con mobiliario suficiente para hacer sus trabajos educativos; generalmente, trabajaba un niño por mesa. Había patio, área de juegos y sanitarios con agua, suficientes para el uso de la comunidad escolar; en el A no se contaba con estos espacios y el acceso al baño era limitado porque era parte de la casa en la que se encontraba.

El A era atendido por un Líder de primer ingreso con licenciatura trunca y realizaba su servicio social en Conafe "como un ejercicio para hacerse más responsable", según la perspectiva de su familia. El B contaba con dos Líderes, una mujer y un hombre, ambos habían trabajado en esa escuela desde el ciclo escolar anterior. Tenían estudios de bachillerato concluido. El chico ingresó con el interés de obtener la beca para continuar sus estudios universitarios, y la líder lo hacía para obtener un apoyo económico.

En ambas escuelas, los materiales didácticos consumibles como colores, hojas y pinturas, que usaban con más frecuencia, resultaban escasos para todos los nińos. También contaban con juegos didácticos, algunos gastados por el uso constante y otros se encontraban incompletos o no contaban con mucha variedad en términos de campos formativos o adecuados a diferentes edades.

En las dos escuelas se disponía de los materiales impresos de los que provee Conafe, las Unidades de Aprendizaje Autónomo (UAA). Sin embargo, dado que las niñas y nińos de preescolar aún no han desarrollado la lectoescritura por completo y el material no ofrecía orientaciones, ni actividades para trabajarlo en este nivel educativo, se encontró que su diseńo era inadecuado para ellos. Por esta razón, sólo eran un material de referencia para la planeación de clases por parte de los Líderes. Por otro lado, únicamente en el preescolar B contaban con algunos libros adicionales a las UAA para los pequeños y con el Libro único para aprender a leer, escribir y contar que servía de apoyo para el aprendizaje de la lectoescritura, lo utilizaban en actividades particulares. 
Hasta el momento, los resultados hablan de algunas inequidades en recursos escolares que se presentan en estas escuelas, que, para el preescolar A, son mayores. Ahora, partiendo de la vinculación entre adaptabilidad y equidad que se estableció entre estos referentes conceptuales, se esperaba que, para que se diera la concreción de este criterio en las escuelas, las prácticas pedagógicas multigrado deberían atender de manera diferenciada las necesidades de aprendizaje de niñas y niños, con relevancia y pertinencia. A continuación se presentan algunos hallazgos al respecto.

La jornada escolar tenía una duración de cinco horas. De las cuales, una estaba dedicada a tomar el desayuno que les llevaban sus madres y a jugar de manera libre. Entre dos y tres horas se asignaban a actividades de enseñanza y aprendizaje, con diferentes grados de implicación en las tareas realizadas. Y alrededor de una hora a actividades rutinarias como pasar lista, lavarse las manos o mantener el orden en el grupo, como dirimir conflictos entre los pequeños o mantenerlos dentro del salón de clases.

En el preescolar A se presentó una tendencia a dedicar la mayor parte del tiempo a las últimas actividades. Durante las observaciones se identificó una vinculación entre el manejo del tiempo en la jornada escolar, con otros aspectos escolares que escapaban de las posibilidades pedagógicas del Líder para reorientar el trabajo en el aula. Comenzando por su escaso conocimiento y experiencia de trabajo docente frente a grupo, la insuficiencia de los materiales didácticos con los que contaba para que todas las niñas y niños trabajaran sobre la misma tarea, hasta el poco espacio disponible para que los pequeños realizaran sus trabajos cómodamente y sin interrumpir a otros compañeros.

Al momento de planificar y desarrollar las actividades educativas para niñas y niños de los preescolares visitados, los LEC se enfrentaron a tres retos principales: los propósitos educativos planteados en los materiales didácticos (UAA) y que requerían ser adecuados al preescolar; los materiales que dieran orientaciones concretas para su enseñanza y el proceso de aprendizaje de niñas y niños, así como los diferentes niveles de apropiación del lenguaje escrito de los pequeños en un aula multigrado. Estos elementos fueron clave para la escasa concreción del criterio de adaptabili$d a d$, a raíz de ellos se presentan algunas reflexiones. 
La planificación de las actividades se desarrollaba en dos momentos: bimestral y diariamente. Realizaban la primera con apoyo de los Capacitadores Tutores (CT) y otros LEC, con la intención de establecer las adecuaciones correspondientes entre los temas que se proponían en las UAA y los objetivos concretos que se iban a trabajar durante ese bimestre en preescolar, así como algunos ejemplos de estrategias didácticas para abordarlos, tenía un carácter general para orientar sus prácticas pedagógicas. De igual manera, cada LEC realizaba una planificación diaria en la que organizaban las actividades específicas que utilizarían para abordar los objetivos y en ella consideraban el avance de los pequeños en clase, sus aprendizajes y su edad.

A través de las bitácoras se identificó que, para realizar las adecuaciones, en algunos casos se tomó como referente el tema que se proponía en las UAA, en otros el contenido de los textos propuestos en las Unidades y también los desafíos que en ellas se planteaban. Esto produjo múltiples interpretaciones sobre aquello que se buscaba que los pequeños aprendieran y se ofrecían pocas orientaciones respecto a cómo establecer la vinculación entre el propósito general que se enunciaba en las UAA y los contenidos a trabajar en preescolar, así como la gradualidad que debían considerar para los pequeños en un aula multigrado. En ambas escuelas los LEC consideraban como un criterio clave para hacer las adecuaciones de preescolar "que las actividades no fueran aburridas". En consecuencia, planteaban juegos, dinámicas o trabajos al aire libre para abordar los contenidos y lograr mantener "activos" a nińas y niños.

Durante las observaciones se identificaron secuencias didácticas similares en ambas escuelas, pero orientadas a diferentes propósitos educativos. Se iniciaba con el pase de lista y alguna canción o dinámica de activación para enfocar la atención de niñas y niños. Se enunciaba la primera actividad o "trabajo" y se explicaba de manera particular cómo la iban a realizar los niños menos y más avanzados en la escritura y la lectura, se daba un momento para realizarla de manera independiente $y$, conforme los niños acababan sus actividades, les daban nuevas. Para los pequeños que tenían mayor dificultad para realizar el trabajo propuesto se les daba más tiempo y se les brindaba apoyo personalizado, ya fuera por parte del LEC o de un compañero más avanzado. 
Generalmente, los trabajos tenían que ver con colorear o ilustrar para los menos avanzados y hacer ejercicios que involucraban escritura (copiar algunas frases cortas o hacer secuencias de trazos o letras) u operaciones básicas para los más avanzados. Dentro del aula no se observó el desarrollo de planas o repetición de operaciones básicas; sin embargo, estas tareas se dejaban para realizarlas en casa. Estas acciones, desde la taxonomía de Anderson y Krathwohl (2001), se relacionan con procesos de baja demanda cognitiva asociados a recordar o reconocer ${ }^{6}$ conocimientos particulares.

$\mathrm{Al}$ mirar las respuestas de las bitácoras en contraste con lo observado, se identificó poca vinculación entre lo planeado y las actividades realizadas pues, al llevarlas a la práctica, solían disminuir la intención cognitiva con la que fueron planteadas. Por ejemplo, en la planeación del líder A estaba realizar un experimento sencillo siguiendo instrucciones (un volcán); esta actividad se ubicaba en el nivel de demanda cognitiva de aplicación, ${ }^{7}$ mayor que las mencionadas anteriormente. Sin embargo, por falta de los materiales adecuados, la actividad propuesta se modificó hacia realizar un dibujo de un volcán que se asocia con las actividades de baja demanda cognitiva.

Dos aspectos resultaron diferentes entre ambas escuelas, una, la atención educativa diferenciada en equidad y relevancia y, dos, la evaluación "personalizada” y formativa. Respecto a la primera, desde la observación y las entrevistas se identificó que más que la capacitación en la implementación en el Modelo ABCD para los Líderes o la proporción de estudiantes por grupo, el conocimiento y la experiencia que ya habían desarrollado los LEC de la escuela $\mathrm{B}$ durante el ańo previo de trabajo con los mismos niños era lo que permitía que pudieran hacer una mejor distinción entre las necesidades de apoyo educativo que requerían sus niñas y niños, para darles atención diferenciada según su progreso de aprendizaje con mayor relevancia, así como tener mejor manejo de grupo para implicarlos en las actividades propuestas.

\footnotetext{
${ }^{6}$ Se refiere a acciones que implican el uso de conocimientos de la memoria, tales como: repetir acciones conocidas, evocar objetos, eventos o hechos, realizar movimientos en forma repetitiva, entre otras (Anderson y Krathwohl, 2001).

${ }^{7}$ Se refiere a la ejecución de situaciones en las que se utilice el material aprendido a través de experimentos, usar un procedimiento en un ejercicio o para resolver un problema (Anderson y Krathwohl, 2001).
} 
Como ya se mencionaba al inicio, para este tipo de escuela los LEC, por norma, no pueden permanecer más de dos ciclos escolares en la misma comunidad, lo que lleva a desaprovechar la experiencia que ya han ganado en el conocimiento de los niños y de formación como docentes.

La evaluación del aprendizaje debería ser personalizada y formativa, según el planteamiento del Modelo ABCD. En el preescolar A el LEC solía establecer charlas particulares con los pequeños para recordar los trabajos realizados, cuestionarlos sobre su comportamiento durante el día o pedirles que repitieran las actividades solicitadas. En el B, se realizaban con menor frecuencia pláticas individuales como se describen en la escuela $\mathrm{A}$, pero se observó que los Líderes se acercaban a diferentes niños mientras hacían sus trabajos y les daban recomendaciones a través de preguntas que les permitían recordar la actividad solicitada, a la vez que obtenían información sobre cómo estaban haciendo la actividad e identificaban la dificultad particular que se les presentaba. Esta manera de desarrollar la evaluación era formativa y ayudó a los niños a terminar sus trabajos con mayor rapidez y de acuerdo con lo que se había solicitado.

En las entrevistas se identificó que estas formas de realizar la evaluación respondían a diferentes concepciones sobre lo que significaba que este proceso fuera "personalizado". Para el líder del preescolar A, se daba al hablar de manera individual con los niños acerca de sus actividades, aunque no les ofreciera retroalimentación sobre cómo mejorar sus trabajos. Mientras que los líderes de la escuela B comentaron que no sabían cómo realizar ese tipo de evaluación, que conforme veían que a los niños se les presentaban dificultades, los apoyaban en particular, concebían la evaluación como un proceso que realizaban al final de los temas desarrollados. Sin embargo, sus prácticas de evaluación eran más cercanas a lo que se entiende como "personalizada y formativa" desde el Modelo pedagógico.

En particular sobre la lectoescritura, es un aprendizaje en el que los padres de familia tenían altas expectativas. Durante las entrevistas se identificó que lo consideraban una ventaja para el ingreso de sus hijos a la primaria, así como una posibilidad para que tuvieran un mejor aprovechamiento académico de ese nivel. 
En este sentido, los padres de familia mencionaron que realizaban ejercicios en casa con los pequeños, adicionales a los que les proponían los LEC de tarea, tales como hacer planas de algunas letras u operaciones básicas.

Por su parte, se identificó que los líderes compartían y buscaban atender estas expectativas de los padres y los propósitos escolares en las aulas, realizando distintas actividades que surgían de su propia experiencia para aprender a leer y escribir, así como de las sugerencias que les ofrecían distintas figuras como Capacitadores, Tutores y otros LEC.

Si bien desde el Modelo ABCD no se identifica un planteamiento específico para el aprendizaje de la lectoescritura en preescolar y primaria, desde el Programa para la Educación Preescolar 2011, que fue tomado como marco para el modelo pedagógico de Conafe, se reconoce que se facilitará el proceso de contacto formal con el lenguaje oral y escrito como herramienta de comunicación y para seguir aprendiendo, en el que niñas y niños 192 adquirirán:

confianza para expresarse, dialogar y conversar en su lengua materna; mejorarán su capacidad de escucha y enriquecerán su lenguaje oral al comunicarse en situaciones variadas. A su vez, desarrollarán interés y gusto por la lectura, usarán diversos tipos de texto y sabrán para qué sirven; se iniciarán en la práctica de la escritura al expresar gráficamente las ideas que quieren comunicar y reconocerán algunas propiedades del sistema de escritura (SEP, 2011, p. 17).

Así se establece de manera explícita el desarrollo del lenguaje oral y escrito en preescolar como el primer eslabón de un proceso que ofrecerá oportunidades para que niñas y niños se acerquen, inicien y enriquezcan sus conocimientos en el mismo, como "sujetos activos y creativos que aprenden por medio de sus propias acciones sobre los objetos del mundo e interactuando con ellos" (Ferreiro y Teberosky, 1979, p. 22).

Desde la perspectiva de Ferreiro:

La escritura puede ser conceptualizada de dos maneras muy diferentes y, según sea el modo en que se le considere, las consecuencias pedagógi- 
cas difieren drásticamente. La escritura puede ser considerada como una representación del lenguaje o como código de transcripción gráfico de las unidades sonoras. La diferencia esencial es la siguiente: en el caso de la codificación ya están predeterminados tanto los elementos como la relaciones, el nuevo código no hace más que encontrar una representación diferente para los mismos elementos y las mismas relaciones; su aprendizaje se concibe como la adquisición de una técnica. Por el contrario, en el caso de la creación de una representación ni los elementos, ni las relaciones están predeterminadas; su aprendizaje se convierte en la apropiación de un nuevo objeto de conocimiento, o sea, en un aprendizaje conceptual (Ferreiro (1997, pp. 13-14).

Al establecer un contraste entre los propósitos educativos que se plantean y lo que Ferreiro postula, pareciera que los primeros conceptualizan el proceso de alfabetización, con base en las características de la representación, más que de la codificación. Lo que desde la adaptabilidad representa el reconocimiento de la experiencia y la construcción particular de significados en torno al lenguaje escrito de cada niña o niño. Esto implica brindar oportunidades de aprendizaje diversas con equidad y relevancia a los pequeños, mientras que desde la pertinencia cultural se reconoce que es un contenido valioso, en tanto se convierte en un medio para seguir aprendiendo.

De igual manera, Ferreiro y Teberosky (1979) mencionan que los primeros intentos de escritura se pueden presentar desde los dos ańos, sin que el propio acto de escribir implique la interpretación de la escritura producida. Desde el punto de vista constructivo, la escritura en niñas y niños sigue una línea de evolución bastante regular en la que se distinguen tres grandes periodos:

1. Distinción entre el modo de la representación icónico y el no icónico; 2. la construcción de formas de diferenciación (control progresivo de las variaciones sobre los ejes cualitativo y cuantitativo); 3 . la fonetización de la escritura (que inicia con un periodo silábico y culmina en el periodo alfabético). En los dos primeros, lo escrito no está regulado por diferencias o semejanzas entre los significantes sonoros. Es la atención a las propiedades sonoras del significante lo que marca el ingreso al tercer gran periodo de evolución (Ferreiro, 1997, p. 20). 
Desde esta perspectiva, se podría pensar que para el aprendizaje de la lectoescritura en un aula multigrado se deberían ofrecer distintas oportunidades, adecuadas al periodo en el que niñas y niños se encuentren, pero con un claro propósito que se oriente hacia el proceso de construcción del aprendizaje, no como una cuestión de métodos, sino de construcción de significados de lo que se entiende por leer y escribir.

Considerando que en las aulas multigrado observadas convivían niñas y niños de entre 2 y 5 años y que el paso por los tres periodos que plantea Ferreiro (1997) se da alrededor de estas edades, se puede reconocer la enorme diversidad en el desarrollo de la escritura que se presentaba en las aulas multigrado observadas, sólo por su edad. Por tanto, su atención pedagógica con equidad $y$ relevancia fue un reto.

Con anterioridad se advertía que las actividades que se proponían a niñas y niños se podrían considerar de baja demanda cognitiva. Al mirarlas a la luz de la conceptualización de los procesos 194 de alfabetización que seńala Ferreiro (1997) y los propósitos de la educación preescolar, se identificó que en ambos preescolares las acciones para el aprendizaje encontraban mayor vinculación con la alfabetización a través de la codificación. Se favorecían las actividades que se centraban en aspectos figurales, relacionados con la calidad y la convencionalidad del trazo (Ferreiro, 1997), tales como copiar frases cortas del pizarrón, remarcar en fotocopias las letras o sílabas que estaban trabajando o colorear dibujos relacionados con las mismas letras. No se identificaron actividades asociadas a la construcción de significados en torno a los procesos de lectoescritura, ni sobre los medios gráficos o sonoros para la representación de significados que tomaran en cuenta el proceso de aprendizaje de niñas y niños.

Es importante resaltar que estas actividades se proponían a aquellos niños que ya tenían algún conocimiento en la lectoescritura, pero para los más pequeños que aún no la aprendían, la oferta era aún más limitada, ya que únicamente se les solicitaba colorear o hacer dibujos como medio de adecuación de los contenidos que se planteaban a los otros. Por otra parte, la investigación reconoce una ausencia importante en términos del desarrollo de la oralidad a través de la expresión, el diálogo y la 
conversación como base para la alfabetización y que se encuentra en los propósitos del preescolar.

\section{CONCLUSIONES}

La adaptabilidad desde su acepción pedagógica en grupos multigrado, enfrentó retos importantes para su concreción en los preescolares observados, pero también aspectos que la favorecían. A continuación se resaltan aquellos que fueron clave para analizarlos.

Al mirar los resultados a la luz de los referentes conceptuales, se reconoce que la adaptabilidad requiere de los criterios de disponibilidad, accesibilidad y aceptabilidad para concretarse en las escuelas, lo que responde a la interdependencia e indivisibilidad entre estos criterios. En este sentido, no se puede lograr el desarrollo pleno de un criterio sin los otros. De igual manera, se reconoce que, si no se cuenta con condiciones iniciales de disponibilidad y accesibilidad en recursos como infraestructura escolar, mobiliario para las aulas, materiales didácticos y docentes con formación profesional adecuada, resulta aún más complicado concretar la aceptabilidad y la adaptabilidad. En los preescolares visitados esto se expresó a través de la inequidad en la existencia de recursos materiales y las limitadas oportunidades de aprendizaje con relevancia y pertinencia que se generaban en ellos, asociadas principalmente a la escasa formación de los Líderes y su acompañamiento.

La atención pedagógica con equidad, relevancia y pertinencia tendientes a la concreción del criterio de adaptabilidad se favoreció en las escuelas en las que el LEC tenía experiencia de trabajo con el mismo grupo desde el ciclo escolar anterior. Resulta fundamental que los LEC tengan mayor experiencia para trabajar con los niños de preescolar, así como contar con acompańamiento a su práctica y materiales que les ofrezcan orientaciones más precisas sobre cómo realizar sus adecuaciones y actividades para el trabajo en aulas multigrado.

Se identificó como una ventaja para la adaptabilidad que los grupos fueran pequeños, permitiendo que el LEC ofreciera atención personalizada a niñas y niños durante sus actividades. 
Si bien los niveles de equidad y relevancia en la atención pedagógica, fueron distintos entre los grupos observados, esta posibilidad representó una importante oportunidad de aprendizaje para todos los pequeńos.

En contraste, se reconoce que, a pesar de que estos grupos contaban con las condiciones grupales y de atención personalizada para desarrollar un proceso pedagógico orientado a la construcción de significados, la escasa formación y conocimiento de los Líderes sobre el desarrollo de niñas y niños en edad preescolar y, específicamente, en el desarrollo de la lectoescritura, influyó para que la equidad y la relevancia de las intervenciones pedagógicas fueran en detrimento, al no identificar las necesidades particulares de apoyo para los niños que se encontraban en diferentes periodos de construcción de este proceso y así poder ofrecer las actividades adecuadas para orientarlos, afectando principalmente a los más pequeńos.

\section{REFERENCIAS BIBLIOGRÁFICAS}

Anderson, L., y Krathwohl, D. (2001). A taxonomy for learning, teaching and Assessing: A revision of Bloom's Taxonomy of Educational Objectives. Nueva York: Longman.

Bassedas, E., Huguet, T., y Sole, I. (2006). Aprender y enseñar en educación infantil (6ta ed.). Barcelona, España: Graó.

Bobbio, N. (1993). Igualdad y libertad. Barcelona: Paidós ICE/UAB.

Bolívar, A. (2005). Equidad educativa y teorías de la justicia. REICE. Revista Iberoamericana sobre Calidad, Eficacia y Cambio en Educación, 3(2), 42 - 69.

Conafe. (2010). Educación Preescolar Comunitaria. México: Conafe.

Conafe. (31 de enero de 2018). Educación comunitaria del Conafe. Recuperado de https://www.gob.mx/conafe/ acciones-y-programas/educacion-comunitaria-del-conafe

Comisión Nacional de los Derechos Humanos (CNDH). (20102017). ¿Qué son los Derechos Humanos? Recuperado de http://www.cndh.org.mx/Que_son_derechos_humanos 
Grupo Europeo de Investigación sobre la Equidad de los Sistemas Educativos (GERESE) (2003). L'équité des systèmes éducatifs européens. Un ensemble d'indicateurs.

Dubet, F. (2005). La escuela de las oportunidades. ¿Qué es una escuela justa? ( $1^{\circ}$ ed.). (M. Polo, Trad.) Barcelona: Gedisa.

Ferreiro, E. (1997). Alfabetización. Teoría y práctica. México: Siglo XXI.

Ferreiro, E. (1999). Vigencia de Piaget. México: Siglo XXI.

Ferreiro, E., y Teberosky, A. (1979). Los sistemas de escritura en el desarrollo del niño. México: Siglo veintiuno.

INEE. (2014). El Derecho a una Educación de Calidad. Informe 2014. México: INEE.

INEE. (2015). El aprendizaje en preescolar en México. Informe de resultado Excale 00 aplicación 2011. Lenguaje y comunicación y Pensamiento matemático. México: INEE.

INEE. (2017a). Panorama Educativo de México 2016. Indicadores del Sistema Educativo Nacional. Educación básica y media superior. México: INEE.

INEE. (2017b). Proyecto Nacional de Evaluación y Mejora Educativa de Escuelas Multigrado (PRONAEME). México: INEE.

Rawls, J. (2003). Teoría de la justicia. México: Fondo de Cultura Económica.

Rodríguez, C.R. (2008). Equidad de la educación en México. Propuesta de un sistema de indicadores. Perspectivas Sociales, 10(2), 55-79.

Santos, L. (2007). Didáctica multigrado: la circulación de los saberes en una propuesta diversificada. Quehacer Educativo, $81,22-32$.

Santos, L. (2011). Aulas multigrado y circulación de los saberes: especificidades didácticas de la escuela rural. Profesorado. Revista de curriculum y formación del profesorado, 15(2), 71-91.

Sen, A. (1987). Equality of What? En Liberty, equality and law. Selected Lectures on Moral Philosophy. Salt Lake City: University of Utah Press. 
SEP. (2011). Programa de estudio 2011. Guía para la educadora. Educación Básica Preescolar. México: SEP.

Tomasevski, K. (2004). El asalto a la educación, Barcelona: Intermon-Oxfam.

Walzer, M. (2001). Las esferas de la justicia: una defensa al pluralismo y a la igualdad. México: Fondo de Cultura Económica. 